\title{
Transfer of visual and haptic maze learning in rats
}

\author{
TUAN D. TRAN, LAURA C ADDUCCI, LIZA D. DEBARTOLO, \\ BETH A. BOWER, and EUGENE R. DELAY \\ Regis University, Denver, Colorado
}

\begin{abstract}
Cross-modal transfer of learning between black/white and rough/smooth discrimination tasks was studied in a two-choice maze procedure in which the stimuli for both discriminations were located on the floor of the maze. Rats were trained initially with cues of one modality and then given transfer training with cues of the second modality. The amount of transfer produced by two criteria of learning, 9 correct responses in 10 trials and 18 correct responses in 20 trials, was also studied. Bidirectional cross-modal transfer of learning was demonstrated more clearly with the more stringent learning criteria. These positive transfer effects appeared primarily to be the result of general transfer processes.
\end{abstract}

During the past 20 years, increased interest in interactions between sensory modalities in nonhuman animals has contributed to greater understanding of perceptual, learning, and memory processes (Ettlinger \& Wilson, 1990). One method particularly suited for studying these interactions is cross-modal transfer (CMT) of learning. When applied to discrimination learning, this involves the transfer of information learned from a task by using cues in one sensory modality to a similar task subsequently learned by using cues in a second modality. One example of this is to train a subject to discriminate between two visual stimuli of different intensities until the subject has reached a stipulated criterion. Then the subject is trained to discriminate between two stimuli of different intensities to a second modality (e.g., auditory). CMT is said to occur when the rate of learning of the second discrimination is faster for this subject than for a control subject that has not received experience with cues in the first modality. In their recent review, Ettlinger and Wilson noted that a number of species are capable of CMT. However, large gaps remain in our ability to demonstrate transfer between specific sensory modalities in many species or to examine the processes associated with these transfer effects.

Over and Mackintosh (1969) were the first to demonstrate CMT in the rat in visual and auditory intensity discrimination tasks. Since then, several studies have elucidated many of the characteristics of CMT processes between these modalities (Church \& Meck, 1983; Delay, 1986; Yeterian, 1977; Yeterian, Waters, \& Wilson, 1976). However, only limited research effort has been directed toward examining the potential for transfer of information between the somatosensory and visual modalities in

This research was supported by NSF Grant BNS-8909803 awarded to E.R.D. Correspondence should be addressed to E. R. Delay, Department of Psychology, Regis University, 3333 Regis Blvd., Denver, CO 80221. rats, and most of this research has focused on bimodal compound conditioning. Apparently, rats can coordinate bimodal information when the task requires them to discriminate between simultaneously or sequentially presented combinations of visual and somatosensory cues. For example, rats are able to discriminate between pairs of compound visuosomatic stimuli that differ in brightness and roughness (Pinto-Hamuy et al., 1987) or that differ in brightness and haptic configuration (LeVere \& LeVere, 1982). However, evidence of transfer has not been as obvious with CMT procedures.

In the only report to date, Denny, Clos, and Rilling (1989) investigated CMT of visual and somatosensory discrimination learning in rats trained in a Y-maze. Six subjects were initially trained with brightness cues in a delayed matching-to-sample task. Although all rats reached criterion on the black/white problem, only one rat showed evidence of transfer to a delayed matchingto-sample discrimination involving smooth and rough (hardware cloth) stimuli. Denny et al. suggested that their tactual matching-to-sample task may have been too complex for most rats to learn and may have masked CMT effects. Moreover, the authors noted that the haptic cues contained an extradimensional visual component that prevented the problem from being exclusively cross-modal. Consequently, the status of CMT between these two modalities in the rat is unclear.

To study visuosomatic CMT, in the present experiment we employed a somewhat simpler procedure by conditioning rats to learn a black/white discrimination and a rough/smooth discrimination in a Y-maze with the discriminative stimuli placed on the floor. In addition, this experiment explored the effects of the learning criterion used during the first discrimination task. Schreurs and Kehoe (1987) found that the amount of CMT seen with a rabbit's nictitating membrane response was directly related to the level of conditioning reached with the first modality. Hence, the effects of two criteria for learning on CMT were examined. The results of the pres- 
ent study indicate that CMT between visual and somatosensory modalities can occur in this paradigm but that it is more effective when the criterion for learning is more stringent.

\section{METHOD}

\section{Subjects}

The subjects were 144 male, naive Sprague-Dawley albino rats which, by the first training session, were between 90 and 120 days old. They were housed singly in their home cages with food available ad lib. All rats were trained during the light portion of their 12:12-h light:dark cycle. The rats were maintained on a 23 -h water deprivation cycle and were handled for 5-10 min each day for at least 3 consecutive days prior to the first training session.

\section{Apparatus}

The rats were trained in a two-arm maze painted black throughout (see Figure 1). The maze had a startbox connected to a $25 \times$ $25 \mathrm{~cm}$ choice area and two adjacent arms, $10 \times 50 \mathrm{~cm}$ long, each ending with a goal area. The floors of the startbox and goal areas were stationary. Either of two removable floors, one for presenting visual stimuli and one for presenting haptic stimuli, could be positioned under the choice area and the maze arms. The visual stimulus floor was made of two sheets of transparent Plexiglas hinged on one side. The floor was divided into two halves by placing black and white stimuli, each extending the length of the choice area and one of the maze arms, between the Plexiglas sheets. For the white stimulus, white translucent paper was illuminated by two rows of $40-\mathrm{W}$ softwhite incandescent lights located beneath the maze. The intensity of these lights was regulated with a rheostat. Light measured $45 \mathrm{~lx}$ at $0.5 \mathrm{~cm}$ above the white half of the floor. The black stimulus was a black nontranslucent paper, and light intensity was between $0.67 \mathrm{~lx}$ at $1 \mathrm{~cm}$ from the edge of the white half and $0.5 \mathrm{~cm}$ above the floor to less than $0.033 \mathrm{~lx}$ at $1 \mathrm{~cm}$ from the outer wall.

The floor for the haptic stimuli also was divided lengthwise into halves by rough and smooth stimuli mounted to the top of brown press board. The haptic stimuli were made from K-Lux Clear Prismatic (PTC-25) fluorescent light cover paneling which had a repeating pattern of peaks and depressions on one side and was smooth on the other side (see Figure 2). During haptic training, a red light, located above the apparatus, provided $6 \mathrm{~lx}$ of reflected light for the experimenter. A water well was located on the floor of the goal area of each arm.

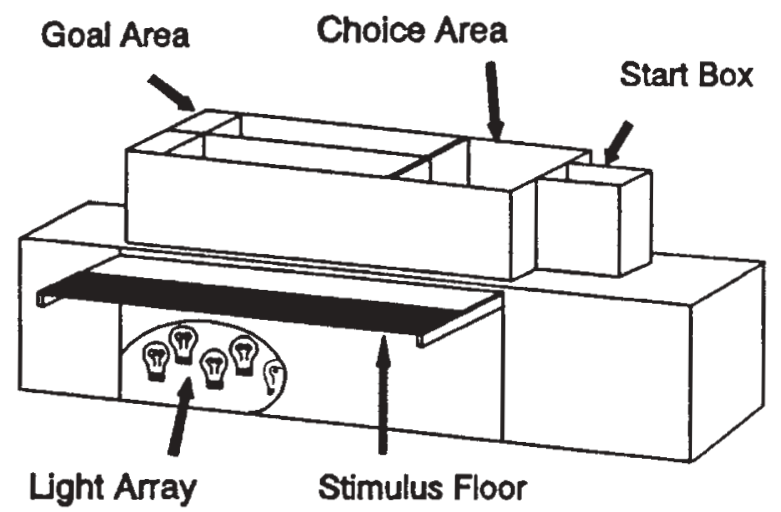

Figure 1. An illustration of the two-choice maze with the stimulus floor (in this case with the visual stimuli) removed from under the maze. A portion of the light array underneath the floor of the maze can be seen in the cutaway.

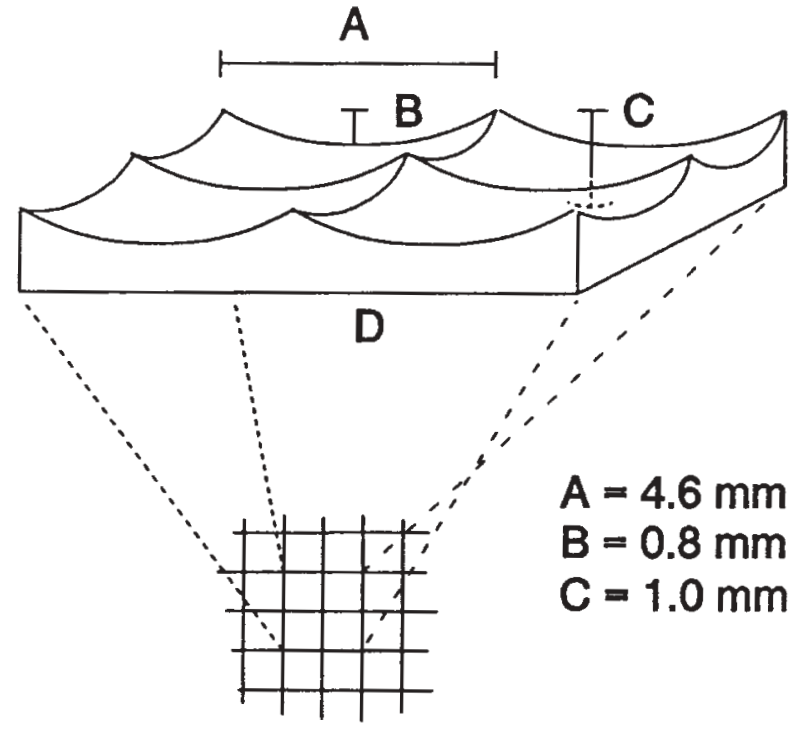

Figure 2. A diagrammatic representation of the acrylic material that served as the rough haptic stimulus. The upper part of the figure is an enlargement of four squares from the repetitive pattern located on one side of the acrylic. The basic configuration of each square was concave with a peak at each corner. The peaks were 4.6 mm apart (A) and were connected by curvilinear ridges. The bottom of each ridge $(B)$ was $0.8 \mathrm{~mm}$ below the tip of the peaks, and the bottom of the arc at the center of the square (C) was $1.0 \mathrm{~mm}$ below the peaks. The opposite side of the acrylic material (D) was a relatively uniform surface which served as the smooth $\mathrm{S}+$. Using the same material for rough and smooth stimuli controlled for olfactory cues.

\section{Procedure}

Each rat explored the maze for at least 10 min on a clear acrylic floor with no stimuli or water reinforcers present on Day 1. On Day 2, each rat was trained to locate the water reinforcer, and on Day 3, each rat was given 20 trials of alternation training. Discrimination training began on Day 4. During discrimination training, each trial began when the door of the startbox was raised to allow the rat to enter the choice area and the maze arms. The water well in each maze arm was hidden behind a stimulus door that matched the floor stimulus. If the rat advanced at least half-way down the $\mathrm{S}+\mathrm{arm}$, the goal area door was removed to give the rat access to $0.15 \mathrm{ml}$ of a $10 \%$ sucrose solution $(\mathrm{w} / \mathrm{v})$ reinforcer and a matching stimulus door was inserted at the entrance of the arm to ensure that the rat remained in the maze arm for $10 \mathrm{sec}$. If the rat entered the $\mathrm{S}-$ arm, the goal area door was not removed, and the matching stimulus door was inserted to force the rat to remain in the arm for a period of $30 \mathrm{sec}$. Also, the $\mathrm{S}+$ and $\mathrm{S}-$ stimuli remained on the same side of the maze during the next trial as a correction procedure.

During initial acquisition training, each rat was randomly assigned to one of four $\mathrm{S}+$ training conditions $(N \mathrm{~S}=32)$ : (1) visualblack stimulus as the $\mathrm{S}+,(2)$ visual-white $\mathrm{S}+$, (3) haptic-rough $\mathrm{S}+$, and (4) haptic-smooth $\mathrm{S}+$. Half the rats in each group were trained until they reached a learning criterion of 9 correct responses out of 10 trials $(9 / 10)$, and the other half were trained until they reached a criterion of 18 correct responses out of $20(18 / 20)$ trials. All trials, including the correction trials, were counted toward criterion. A random sequence for locating each $\mathrm{S}+$ and $\mathrm{S}-$ during each trial was determined prior to each training session. To prevent the development of a side preference, the S+ could not be located on the same side on more than 3 consecutive trials, unless the correction procedure for erroneous responses was used. Each rat was given 20 trials per day during a single training session, 
fewer if it reached criterion during the session. The interval between trials ranged from 30 to $60 \mathrm{sec}$. The training session for each rat lasted about $0.5 \mathrm{~h}$, after which the rat was given access to a water bottle in the home cage for $0.5 \mathrm{~h}$.

After the rat reached its assigned criterion, the rat was maintained on the 23-h deprivation schedule for 3 days before it was given transfer training. Transfer training involved the same procedures, except that the cues of the opposite modality were used. Half the rats in each $\mathrm{S}+$ condition were trained with one of the $\mathrm{S}+\mathrm{s}$ in the opposite modality and the rest were trained with the other $\mathrm{S}+$. For example, half the rats initially trained with the white $\mathrm{S}+$ were given transfer training with the rough $\mathrm{S}+$ and half with the smooth $\mathrm{S}+$. Transfer training for each rat was to the same learning criterion as initial training. Although albino rats have minimal visual sensitivity for long wavelengths (Green, 1971), the spectral sensitivity to the red light was unknown. Therefore, after reaching criterion on the haptic discrimination, 4 rats from the $9 / 10$ group and 4 from the 18/20 group were given two additional haptic trials in complete darkness to determine whether the rats were solving this task with the aid of the red light. Fifteen of 16 responses were to the correct arm. The rat with the incorrect response was given one additional trial, which it performed correctly.

To determine whether exposure to the discrimination cues per se, habituation to the apparatus and training procedures, or other nondiscrimination variables might have an effect during transfer training, an additional 16 rats were given pseudodiscrimination training. This training began on Day 4 and continued for three sessions (60 trials), the most required by any of the rats given standard discrimination training to reach criterion during initial acquisition. Pseudodiscrimination training was identical to standard discrimination training, except that the locations of the stimuli and the reinforcer were varied independently of each other. The stimuli were randomly assigned to each arm of the maze and could be assigned to the same arms for as many as 5 consecutive trials. The location of the reinforcer was randomly assigned also, but it could not be obtained in the same arm for more than 3 consecutive trials. If the rat did not enter the maze arm in which the reinforcer was located, the rat was forced to stay in that arm for $30 \mathrm{sec}$. In addition, the reinforcer was automatically located in the same arm on the next trial, but the stimulus cues were free to vary according to the preassigned sequence. Correct responses and errors were monitored for greater than chance pairing between the stimuli and the reinforcer. (No evidence for this was observed.) Eight of the rats were given pseudodiscrimination training with the visual stimuli. Three days later, 4 of these rats were tested on the standard haptic discrimination with the rough $\mathrm{S}+$ and the other 4 were tested with the smooth $\mathrm{S}+$. Of the remaining 8 rats given pseudodiscrimination training with the haptic stimuli, 4 were tested on the standard visual discrimination with the white $\mathrm{S}+$ and 4 with the black $\mathrm{S}+$ All of these rats were run to the $9 / 10$ criterion.

\section{RESULTS}

Each score, representing the number of trials to criterion, was adjusted before analysis by subtracting either 10 or 20 , the value of the learning criterion for that subject. The scores for initial acquisition training were subjected to an analysis of variance (ANOVA) with criteria (2) and $\mathrm{S}+$ cues (4) as between-subjects variables. This analysis showed that the main effect of criteria was not significant $(F<1.0)$ but that there was a significant difference in scores resulting from the $\mathrm{S}+$ stimulus used during training $[F(3,120)=4.68, p<.005]$. A NewmanKeuls post hoc test $(p<.05)$ indicated that the rats learned the black $\mathrm{S}+$ discrimination in significantly fewer trials than did rats trained with the smooth $\mathrm{S}+$.
The mean trials to criterion for the other $\mathrm{S}+$ groups were intermediate to these groups and did not differ from them. The interaction of criteria and $\mathrm{S}+$ cue approached but did not reach significance $(p=.08)$.

To examine the effects of learning criteria and of initial acquisition training on subsequent transfer testing, the scores for all rats trained with a particular S+ during acquisition were compared with the scores of the rats trained with the same $\mathrm{S}+$ during transfer. For example, a 2 (learning criteria) $\times 3$ (training conditions) betweensubjects ANOVA was used to compare the scores of rats initially trained with the smooth $\mathrm{S}+$ with the scores of the rats trained with the smooth $\mathrm{S}+$ during transfer after receiving initial acquisition with either the white or the black $\mathrm{S}+$. This analysis indicated significant group differences due to learning criteria $[F(1,58)=21.72, p<$ $.001]$ and training conditions $[F(2,58)=6.81, p<.001]$, as well as a significant interaction between the two variables $[F(2,58)=3.69, p<.05]$ (see Figure 3). Separate analyses of the data obtained under each criterion of learning indicated that rats initially trained to the $9 / 10$ criterion with the visual stimuli did not show any transfer to the haptic cues $(F<1.0)$, whereas rats initially trained to the $18 / 20$ criterion did $[F(2,29)=17.47, p<$ $.001]$. Newman-Keuls comparisons $(p<.05)$ revealed that rats initially trained to $18 / 20$ with the black S+ learned the smooth $\mathrm{S}+$ discrimination during transfer in significantly fewer trials than did those initially trained with the white S+. Furthermore, both of these groups learned the smooth $\mathrm{S}+$ discrimination in significantly fewer trials than the rats initially trained with the smooth $\mathrm{S}+$. Similarly, transfer to the rough $\mathrm{S}+$ was also significantly affected by criteria $[F(1,58)=33.87, p<.001]$, training conditions $[F(2,58)=8.70, p<.001]$, and their interaction $[F(2,58)=8.26, p<.001]$. Again, rats initially trained with the visual stimuli to the $9 / 10$ criterion did not exhibit transfer $(F<1.0)$, whereas rats initially trained to the $18 / 20$ criterion showed positive transfer to

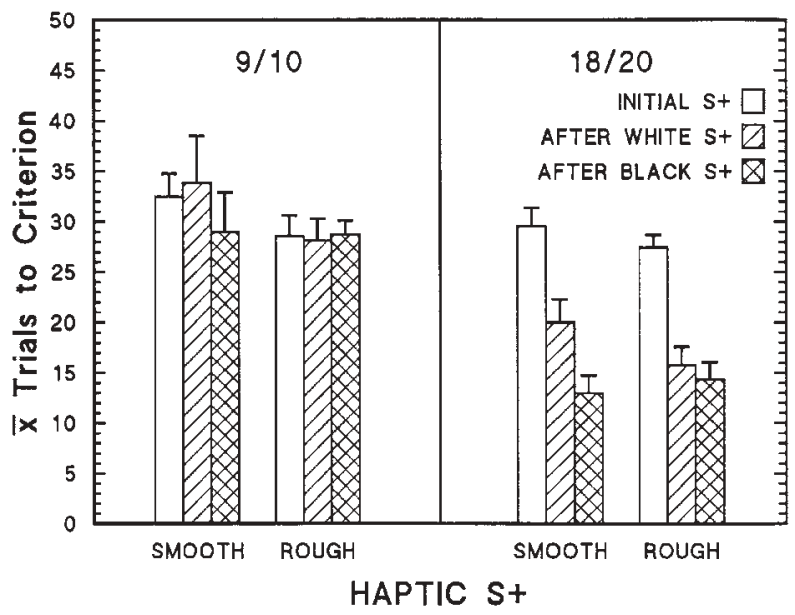

Figure 3. Mean trials to criterion, less the criterion trials, for the rats trained with the haptic stimuli during initial acquisition and in transfer testing after visual training with either the white or the black S+. (Error bars show SEM.) 


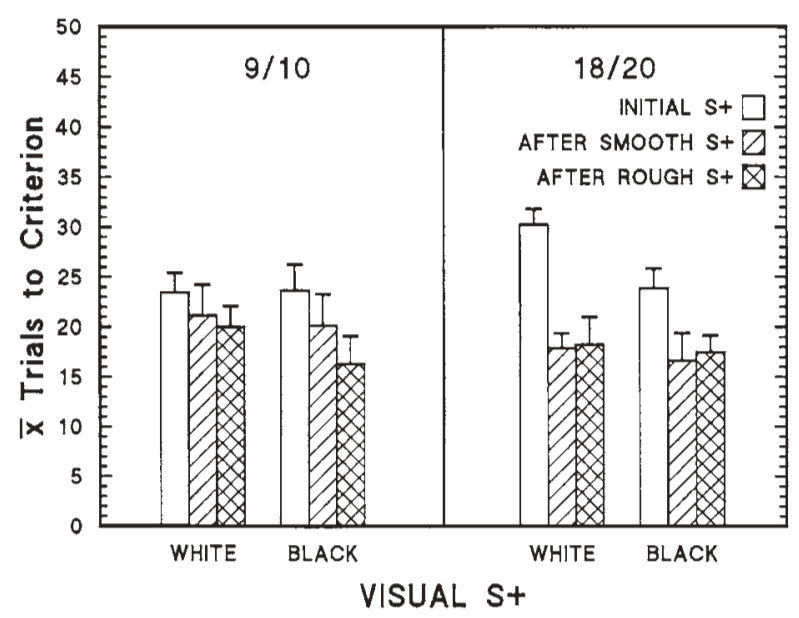

Figure 4. Mean trials to criterion, less the criterion trials, for the rats trained with the visual stimuli during initial acquisition and during transfer testing after haptic training with either the smooth or the rough $\mathrm{S}+$. (Error bars show SEM.)

the rough $\mathrm{S}+$ discrimination $[F(2,29)=24.51, p<$ .001]. In this case, however, transfer was equivalent after initial training with either the white or the black S+.

Positive transfer to visual cues was also seen for rats initially trained with the haptic cues (see Figure 4). Specifically, significant overall transfer was observed to the white $\mathrm{S}+[F(2,58)=9.14, p<.001]$, and there was a significant interaction between training conditions and learning criteria $[F(2,58)=3.53, p<.05]$. Analysis of the scores for rats trained to the $9 / 10$ criterion did not detect any transfer to the white $\mathrm{S}+(F<1.0)$, whereas the analyses of the scores for the rats trained to the 18/20 criterion revealed significant positive transfer $[F(2,29)=$ $15.21, p<.001]$. The latter effect was comparable after training with either the rough or the smooth S+. Finally, the analysis of the data for rats that transferred to the black $\mathrm{S}+$ discrimination indicated a significant effect of training conditions $[F(2,58)=4.15, p<.025]$. Post hoc testing showed that positive transfer to the black S+ task occurred under both learning criteria after training with either haptic cue.

It was possible for rats trained to $18 / 20$ during acquisition to reach a $9 / 10$ criterion during transfer testing at a rate different from rats trained to $9 / 10$ during acquisition. Therefore, 2 (criteria) $\times 2(\mathrm{~S}+)$ between-subjects ANOVAs were used to compare the $9 / 10$ criterion scores for the groups tested with each S+ (see Figure 5). During haptic transfer tests, the rats trained to $18 / 20$ during acquisition reached the $9 / 10$ criterion with the rough $\mathrm{S}+$ during transfer tests significantly faster than the rats trained to the $9 / 10$ criterion during acquisition $[F(1,28)=$ $48.95, p<.001]$. Similarly, regardless of the visual $\mathrm{S}+$ used during acquisition training, the rats initially trained to $18 / 20$ reached the $9 / 10$ criterion during transfer testing with the smooth $\mathrm{S}+$ in significantly fewer trials than did the rats initially trained to $9 / 10[F(1,28)=17.15$, $p<.001]$. During transfer tests with the white $\mathrm{S}+$, rats trained to the $18 / 20$ criterion reached a $9 / 10$ criterion in significantly fewer trials than did the rats conditioned to the $9 / 10$ criterion during acquisition $[F(1,28)=17.15$, $p<.001]$. Transfer scores were similar after training with either the rough or the smooth haptic cue. On the other hand, neither learning criterion nor type of haptic cue used in training had a differential effect on transfer to the black $\mathrm{S}+[F(1,28)=2.71, p>.05]$. However, the $9 / 10$ transfer test scores of the rats trained to $18 / 20$ were significantly lower than the initial acquisition scores of the rats trained to $9 / 10$ on the black $\mathrm{S}+[F(2,29)=4.16$, $p<.05$ ] (see Figure 4).

A between-subjects ANOVA of trials to criterion for the various pseudodiscrimination groups during transfer did not reveal any significant differences. Additional analyses revealed that the pseudodiscrimination rats required significantly more trials to reach criterion than did the rats trained with the standard procedures $[F(1,72)$ $=7.31, p<.01]$, but the interaction was not significant. In addition, separate one-way ANOVAs were used to compare the scores of the pseudodiscrimination groups with their corresponding $\mathrm{S}+$ transfer groups trained with each learning criterion. No significant differences were found when the scores of the pseudodiscrimination rats tested on the visual transfer problem were compared with the visual transfer scores of rats conditioned to the $9 / 10$ criterion with haptic cues during acquisition. However, the scores for these pseudodiscrimination rats were significantly higher than scores at the $9 / 10[F(5,34)=$ $4.45, p<.005]$ and $18 / 20[F(5,34)=3.085, p<.025]$ criteria of visual transfer testing for rats trained to $18 / 20$ during acquisition. Similarly, no significant differences were found between the scores of the pseudodiscrimination rats tested with haptic cues and rats trained to the $9 / 10$ criterion in the haptic transfer tests. In contrast, the scores for these pseudodiscrimination rats were significantly greater than the scores of the standard discrimina-

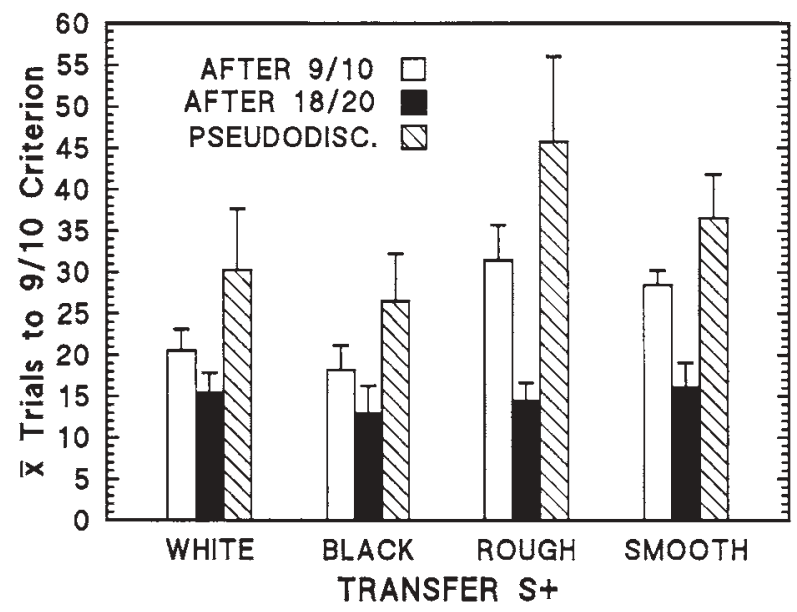

Figure 5. Mean trials to a 9/10 criterion, less the criterion trials, during transfer testing on each $\mathrm{S}+$ for rats receiving initial discrimination training to $9 / 10$ criterion, $18 / 20$ criterion, or pseudodiscrimination training during acquisition. (Error bars show SEM.) 
tion rats trained to the $9 / 10[F(5,34)=12.32, p<.001]$ and the $18 / 20[F(5,34)=15.59, p<.001]$ criteria in the haptic transfer conditions.

In summary, positive transfer between visual and haptic cues was detected when the learning criterion during initial acquisition was the more stringent $18 / 20$. The amount of transfer was similar for most stimuli, except in rats tested on the smooth $\mathrm{S}+$ discrimination task. These rats exhibited better transfer after training with the black $\mathrm{S}+$ than after training with the white $\mathrm{S}+$. Furthermore, the results of the pseudodiscrimination group showed that exposure to the training stimuli per se, the apparatus, etc., could not account for all of the cross-modal transfer observed in the standard discrimination groups.

\section{DISCUSSION}

The scores obtained during initial acquisition training indicated that although the rats were able to learn both visual and haptic discrimination tasks in relatively few trials, the black $\mathrm{S}+$ discrimination was learned more quickly than the smooth $\mathrm{S}+$ discrimination. Also, the results of the rats tested in total darkness indicated that the haptic discrimination was based on somatosensory input and did not require some form of visual cue.

The positive transfer effects seen during testing were particularly interesting because they are the first clear evidence of bidirectional CMT between the visual and somatosensory modalities in the rat. CMT between these two modalities has been demonstrated for other species (see Ettlinger \& Wilson, 1990, for a review). In the rat, CMT of intensity and of temporal discrimination learning between the visual and auditory modalities is well documented (Church \& Meck, 1983; Over \& Mackintosh, 1969; Rudolph \& Delay, 1993). By contrast, interactions between the visual and somatosensory systems has been suggested primarily by conditioning experiments in which visual and haptic stimuli were presented simultaneously (e.g., LeVere \& LeVere, 1982; PintoHamuy et al., 1987). In the only previous study to explicitly examine CMT between these two modalities in rats (Denny et al., 1989), only 1 of 6 rats showed evidence of transfer from visual to haptic stimuli in a matchingto-sample task and a 2nd rat showed some transfer in the opposite direction. Delayed matching-to-sample tasks are more difficult for rats to learn than the two-choice discrimination task used in this study, and it may be that CMT is more apparent in simpler discrimination tasks. However, it should be noted that pilot studies in which the visual stimuli were presented on doors at the entrance to the maze arms were unable to reliably produce evidence of CMT. Locating the stimuli of both tasks on the floor of the maze, and thus focusing the attention of the rat on that part of the maze, also may have been a key to detecting CMT effects.

Clearly, the strength of the transfer effects in this study depended upon the criterion of learning. This finding is consistent with phenomena such as the overlearning reversal effect, in which positive transfer is strength- ened by additional exposure to the discrimination task (cf. Mackintosh, 1969), and with a previous report on a conditioned nictitating membrane response in the rabbit, which showed that the amount of CMT was a direct function of the number of conditioning trials administered with the first modality (Schreurs \& Kehoe, 1987). In the present study, rats trained to the $9 / 10$ criterion, a criterion frequently used in two-choice maze discrimination studies, exhibited only weak transfer from the two haptic conditions to the black $\mathrm{S}+$. On the other hand, when the rats were trained to the $18 / 20$ criterion during acquisition, CMT was evident in all transfer test conditions, independently of whether the criterion during testing was $9 / 10$ or $18 / 20$. The criterion scores of the rats initially given pseudodiscrimination training suggest that the improved performance of the rats trained to the $18 / 20$ criterion was not related to greater habituation or to experience with other nondiscriminative variables. Since the higher criterion did not increase initial acquisition scores, except possibly when training involved the white $\mathrm{S}+$ (cf. Figure 4), the criterion trials probably served as practice trials in which CMT could develop more fully.

One of the primary sources of CMT involves general transfer of information from the first to the second modality. General transfer effects are often attributed to stimulus generalization (Kehoe, 1988), to the development of a perceptual set and/or a response set (Gibson, 1969), or to some form of supraordinate learning that occurs during acquisition of the discrimination task with the first modality (Church \& Meck, 1983). Typically, this type of transfer has comparable effects on the performance of the transfer task, regardless of the specific stimulus attributes relevant to the solution to the discrimination (Bryant, 1968). The equivalence of the scores seen in the transfer sessions for the white, black, and rough $\mathrm{S}+$ after training with the cues of the opposite modality suggests most of the positive transfer seen in this experiment was the product of general transfer processes. Since the visual discrimination was based on a difference in stimulus intensity, whereas the somatosensory discrimination was assumed to be based on the three-dimensional characteristics of the haptic stimuli, transfer may have been related to extradimensional processes such as an increase in attentional focus towards stimuli located on the floor and/or to response-reinforcement relationships. Others have identified similar sources of general transfer effects in cross-modal discrimination tasks (e.g., Clark \& Delay, 1991; Holt \& Kehoe, 1985; Kehoe, 1988; Thomas, Miller, \& Svinicki, 1971). An unexpected finding was that initial training with the white $\mathrm{S}+$ produced significantly less positive transfer to the smooth $\mathrm{S}+$ than did initial training with the black $\mathrm{S}+$ for rats trained to $18 / 20$. It is unclear whether the white $\mathrm{S}+$ rats simply did not develop general transfer processes as fully as the other rats, or whether some other factor influenced their performance during the transfer session. To explore this finding will require additional research. 
The results of this experiment demonstrate bidirectional CMT between the visual and somatosensory modalities in the rat with a two-choice maze discrimination procedure. It appears, however, that the criterion for learning must be stringent enough to provide sufficient practice with the discrimination task before transfer effects can be detected. These findings may be particularly relevant to research examining the neurological basis of visuosomatosensory transfer or of recovery of function. In other research, postoperative auditory CMT training has been found to facilitate recovery of a preoperatively acquired brightness discrimination after injury to the visual cortex (Clark \& Delay, 1991; Delay, 1988). The results of this study suggest that recovery of function research can now be extended to examine the effects of CMT between visual and somatosensory modalities on behavioral recovery after brain injury.

\section{REFERENCES}

Bryant, P. E. (1968). Comments on the design of developmental studies of cross-modal matching and cross-modal transfer. Cortex, 4, 127-137.

Church, R. M., \& Meck, W. H. (1983). Acquisition and cross-modal transfer of classification rules for temporal intervals. In M. L. Commons, R. J. Herrnstein, \& A. R. Wagner (Eds.), Qualitative analyses of behavior: Discrimination processes (Vol. 4, pp. 75-97). Cambridge, MA: Ballinger.

Clark, R. E., \& Delay, E. R. (1991). Reduction of lesion-induced deficits in visual reversal learning following cross-modal training. Restorative Neurology \& Neuroscience, 3, 247-255.

Delay, E. R. (1986). Effects of cross-modal transfer on direct and reversal learning in the rat. Animal Learning \& Behavior, 14, 427-434.

Delay, E. R. (1988). Facilitative effects of cross-modality training on recovery of a conditioned avoidance response following striate cortex ablations in the rat. Neuropsychologia, 26, 661-671.

Denny, M. R., Clos, C., \& Rilling, M. (1989). Delayed matchingto-sample in rats in a Y-maze: Instances of facilitation and immediate cross-modal transfer. Bulletin of the Psychonomic Society, 27, 141-144.
Ettlinger, G., \& Wilson, W. A. (1990). Cross-modal performance: Behavioural processes, phylogenetic considerations and neural mechanisms. Behavioural Brain Research, 40, 169-192.

Gibson, E. J. (1969). Principles of perceptual learning and development. Englewood Cliffs, NJ: Prentice-Hall.

Green, D. G. (1971). Light adaptation in the rat retina: Evidence for two receptor mechanisms. Science, 174, 598-600.

Holt, P. E., \& KeHoE, E. J. (1985). Cross-modal transfer as a function of similarities between training tasks in classical conditioning of the rabbit. Animal Learning \& Behavior, 13, 51-59.

KeHOE, E. J. (1988). A layered network model of associative learning: Learning to learn and configuration. Psychological Review, 95, 411433.

LeVere, N. D., \& LeVere, T. E. (1982). Recovery of function after brain damage: Support for the compensation theory of the behavioral deficit. Physiological Psychology, 10, 165-174.

MacKINTOSH, N. J. (1969). Further analysis of the overtraining reversal effect. Journal of Comparative \& Physiological Psychology Monographs, 67 (2, Pt. 2), 1-18.

Over, R., \& Mackintosh, N. J. (1969). Cross-modal transfer of intensity discrimination by rats. Nature, 224, 918-919.

Pinto-Hamuy, T., Olavarria, J., Guic-Robles, E., Morgues, M., NAssal, O., \& Petit, D. (1987). Rats with lesions in anteromedial extrastriate cortex fail to learn a visuosomatic conditional response. Behavioural Brain Research, 25, 221-231.

Rudolph, T. M., \& Delay, E. R. (1993). Recovery of a photic rate discrimination in rats with visual cortex lesions. Behavioural Brain Research, 53, 189-199.

Thomas, D. R., Miller, J. T., \& Svinicki, J. G. (1971). Nonspecific transfer effects of discrimination training in the rat. Journal of Comparative \& Physiological Psychology, 74, 96-101.

Schreurs, B. G., \& KeHoE, E. J. (1987). Cross-modal transfer as a function of initial training level in classical conditioning with the rabbit. Animal Learning \& Behavior, 15, 47-54.

YeTERIAN, E. H. (1977). The effects of visual or auditory cortical lesions on specific cross-modal transfer in the rat. Neuropsychologia, 15, 517-527.

Yeterian, E. H., Waters, R. S., \& Wilson, W. A. (1976). Posterior cortical lesions and specific cross-modal transfer in the rat. Physiological Psychology, 4, 281-284.

(Manuscript received September 1, 1993; revision accepted for publication March 17, 1994.) 